\title{
Spin transport in fully hexagonal boron nitride encapsulated graphene
}

\author{
M. Gurram, ${ }^{1, *}$ S. Omar, ${ }^{1}$ S. Zihlmann, ${ }^{2}$ P. Makk, ${ }^{2}$ C. Schönenberger, ${ }^{2}$ and B. J. van Wees ${ }^{1}$ \\ ${ }^{1}$ Physics of Nanodevices, Zernike Institute for Advanced Materials, University of Groningen, The Netherlands \\ ${ }^{2}$ Department of Physics, University of Basel, Basel, Switzerland
}

(Received 14 January 2016; revised manuscript received 29 February 2016; published 29 March 2016)

\begin{abstract}
We study fully hexagonal boron nitride $(\mathrm{hBN})$ encapsulated graphene spin valve devices at room temperature. The device consists of a graphene channel encapsulated between two crystalline hBN flakes: thick-hBN flake as a bottom gate dielectric substrate which masks the charge impurities from $\mathrm{SiO}_{2} / \mathrm{Si}$ substrate and single-layer thin-hBN flake as a tunnel barrier. Full encapsulation prevents the graphene from coming in contact with any polymer/chemical during the lithography and thus gives homogeneous charge and spin transport properties across different regions of the encapsulated graphene. Further, even with the multiple electrodes in-between the injection and the detection electrodes which are in conductivity mismatch regime, we observe spin transport over 12.5- $\mu \mathrm{m}$-long distance under the thin-hBN encapsulated graphene channel, demonstrating the clean interface and the pinhole-free nature of the thin $\mathrm{hBN}$ as an efficient tunnel barrier.
\end{abstract}

DOI: 10.1103/PhysRevB.93.115441

\section{INTRODUCTION}

Graphene is considered as an ideal material for spintronics due to its low intrinsic spin-orbit coupling, small hyperfine interaction, tunable carrier density, and high electron mobility [1-3] even at room temperature, which is important for future applications. Theoretically, it is estimated to show long spin relaxation length $\left(\lambda_{s}\right)$ of $100 \mu \mathrm{m}$ and high spin relaxation time $\left(\tau_{s}\right)$ of $100 \mathrm{~ns}[4,5]$. At the moment, experimentally these values are reported up to $\lambda_{s}=30.5 \mu \mathrm{m}$ and $\tau_{s}=12.6 \mathrm{~ns}$ [6]. In a quest to figure out the sources limiting the intrinsic spin transport in graphene, there have been several experiments, which suggest that the role of underlying substrate and the quality of tunnel barrier is crucial [7].

The quality of substrate plays an important role in determining the charge and the spin transport characteristics of graphene. An atomically flat hexagonal boron nitride (hBN) substrate has shown to provide a good base for high mobility graphene [8] and long spin relaxation length [9] because hBN has less charged impurities and the thickness of the bottom $\mathrm{hBN}$ moves the graphene away from the charged impurities in the $\mathrm{SiO}_{2}$ substrate. Also, the effect of the electron-hole puddles can be different for different substrates and it is lower with a hBN substrate [10].

Recently, Kretinin et al. [11] demonstrated the high electronic quality of graphene by encapsulating it from surroundings with different two-dimensional (2D) crystals such as hBN and transition-metal dichalcogenide (TMD). However, this device geometry comprised of one-dimensional edge electrodes [8] with a thick semiconducting TMD top layer which is not compatible for standard four-probe nonlocal spin transport measurements. In this work, we replace the TMD with an atomically thin-hBN flake, which serves two purposes. First, it acts as an encapsulating layer. Second, it acts as a tunnel barrier replacing the conventional oxide tunnel barrier. Therefore, it allows to probe the charge and the spin transport properties of the encapsulated graphene with multiple ferromagnetic (FM) electrodes.

\footnotetext{
*Corresponding author: m.gurram @ rug.nl
}

The previous studies have shown that the graphene interface with spin-sensitive ferromagnetic electrodes affects the spin transport behavior. Further, the conventional oxide tunnel barriers used in spintronic devices suffer from pinholes, inhomogeneous coverage, nonuniform growth, and consequently contributing to the low spin transport properties [7,12]. A 2D layer of thin $\mathrm{hBN}$ with close lattice match to graphene has been shown to exhibit pinhole-free tunneling characteristics [13,14]. It is also predicted to enhance the spin injection efficiency from a ferromagnet into graphene [15].

Yamaguchi et al. [16] first reported spin injection through exfoliated monolayer hBN tunnel barrier into bilayer graphene resulting in quite low spin transport parameters $\left(\tau_{s}=56 \mathrm{ps}\right.$, $\left.D_{s}=0.034 \mathrm{~m}^{2} / \mathrm{s}, \lambda_{s}=1.35 \mu \mathrm{m}\right)$ and low mobility $(\mu=$ $2700 \mathrm{~cm}^{2} / \mathrm{Vs}$ ) of graphene. Further, Kamalakar et al. [17] and $\mathrm{Fu}$ et al. [18] used large-scale chemical vapor deposited (CVD) bilayer hBN tunnel barriers for spin injection into graphene. The spin polarization increased with CVD hBN barriers. However, the quality of graphene channel is limited by the wet transfer technique used for transferring CVD hBN on to graphene. In contrast, we use a dry pickup and transfer technique [19] in order to completely cover the underlying graphene with a thin-hBN flake, which acts a tunnel barrier and also shields the graphene from external polymers and chemicals during the lithography process.

It is also worth discussing here that the previous reports on spin transport in hBN encapsulated graphene are quite promising in terms of improved spin transport parameters [20,21]. However, extracting the correct spin transport parameters is not straightforward due to inhomogeneous spin transport behavior of the hBN encapsulated and the non-hBN encapsulated graphene regions. A similar behavior is also observed for partly suspended high mobility graphene [22]. Using our device geometry, we can achieve more homogeneous charge and spin transport behavior in the graphene channel compared to the previously reported results.

In this work, we report a fully hBN encapsulated graphene spintronics device to overcome the three aforementioned challenges, namely, (1) the influence of underlying substrate, (2) the influence of tunnel barrier interface, and (3) the inhomogeneity in graphene channel. We use a dry pickup 
technique which prevents the graphene from external doping and results in more homogeneous charge and spin transport parameters at room temperature.

\section{DEVICE FABRICATION}

The $\mathrm{hBN} / \mathrm{graphene} / \mathrm{hBN}$ stack is prepared following the dry pickup and transfer technique $[8,19]$. The graphene and hBN flakes were obtained by the exfoliation of highly oriented pyrolytic graphite (HOPG, SPI Supplies, ZYA grade) and hBN powder (HQ graphene). A 90-nm-thick $\mathrm{SiO}_{2} / \mathrm{Si}$ substrate is used for exfoliation and identification of graphene and thin$\mathrm{hBN}$ flakes as it gives a good optical contrast to search for thinner graphene [23] and hBN [24] flakes down to a monolayer.

A polydimethylsiloxane (PDMS) polymer stamp prepared with polycarbonate (PC) layer is used to pick up the flakes. At first, a glass substrate with PDMS/PC is used to pick up a thick top-hBN flake which is used to pick up the thin-hBN flake followed by picking up the graphene flake. Then, the $\mathrm{PC} /$ top-hBN/thin-hBN/graphene stack is released onto a thick bottom $\mathrm{hBN}$ on a $\mathrm{SiO}_{2}(300 \mathrm{~nm}) / \mathrm{Si}$ substrate by melting the PC layer. Next, the PC layer is dissolved in chloroform for $5 \mathrm{~h}$ at $50^{\circ} \mathrm{C}$, followed by annealing in $\mathrm{Ar} / \mathrm{H}_{2}$ atmosphere at $350^{\circ} \mathrm{C}$ for $12 \mathrm{~h}$ to remove the leftover $\mathrm{PC}$ residues on top of the thin $\mathrm{hBN}$ and the top hBN. It is important to note that the graphene beneath the thin $\mathrm{hBN}$ does not come in contact with polymers during the fabrication due to the full encapsulation by hBN flakes.

Thereafter, electron-beam lithography is used for patterning of electrodes on the poly(methylmethacrylate) (PMMA) coated stack followed by electron-beam evaporation to deposit $65 \mathrm{~nm}$ of ferromagnetic cobalt (Co) for spin-sensitive electrodes. Cobalt is capped with 4-nm-thick aluminum (Al) layer to prevent it from oxidation. A schematic of the layer-by-layer device structure is shown in Fig. 1(a).

An optical micrograph of the fabricated device is shown in Fig. 1(b) where the graphene and the thin-hBN flakes are outlined with the black and the white dashed lines, respectively. Due to a slight misalignment during the pickup process, a region of $0.1-0.2 \mu \mathrm{m}$ width along the top edge of the graphene flake is not covered by the thin hBN. However, the electrodes in-between E2 and E5 are deposited on top of the thin-hBN layer, avoiding the uncovered top-edge graphene region. The thin-hBN encapsulated graphene does not come in contact with polymers whereas the graphene regions which are not covered by the thin hBN are touched by PC and PMMA polymers during the fabrication.

We performed atomic force microscopy (AFM) imaging to find the thickness of each flake used in the device fabrication. It is found to be $0.40 \mathrm{~nm}$ for graphene and $0.52 \mathrm{~nm}$ for thin-hBN layer. These values are in close agreement with the experimentally reported thickness for single-layer (1L)graphene [25] and 1L-hBN [14,17].

\section{RESULTS AND DISCUSSION}

We report the measurements for the different regions of fully encapsulated graphene channel as labeled in the optical image shown in Fig. 1(b). Specifically, Ch1 $(4.5 \mu \mathrm{m}$, between $\mathrm{E} 2$ and $\mathrm{E} 3)$ and $\mathrm{Ch} 3(3 \mu \mathrm{m}$, between $\mathrm{E} 4$ and $\mathrm{E} 5)$ regions consist of graphene encapsulated by thin hBN. Ch2 $(5 \mu \mathrm{m}$, between

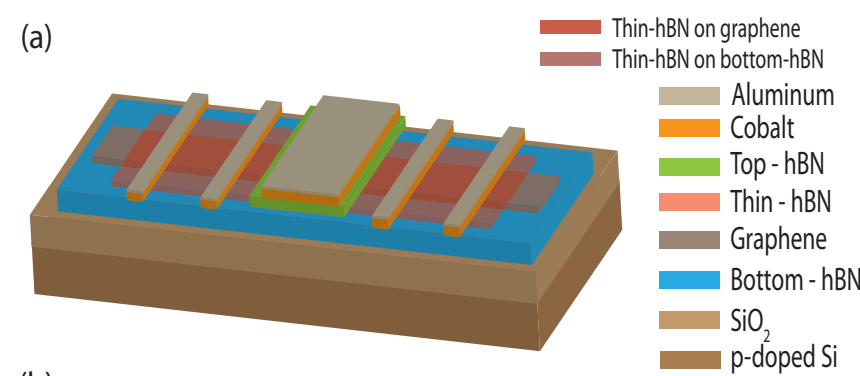

(b)

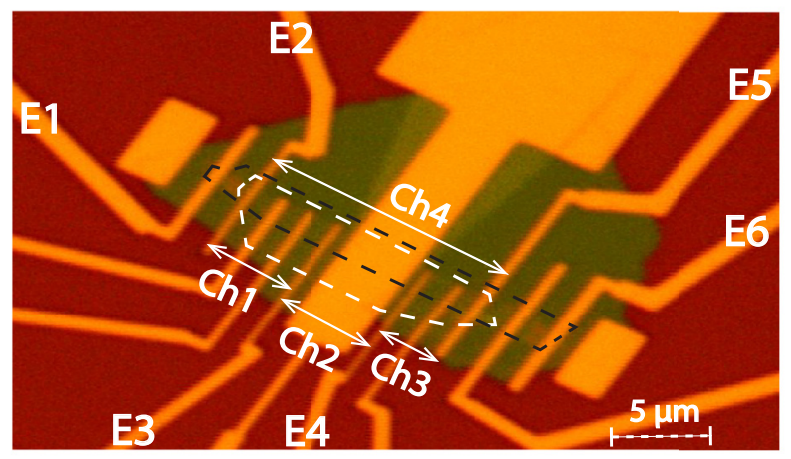

(c)

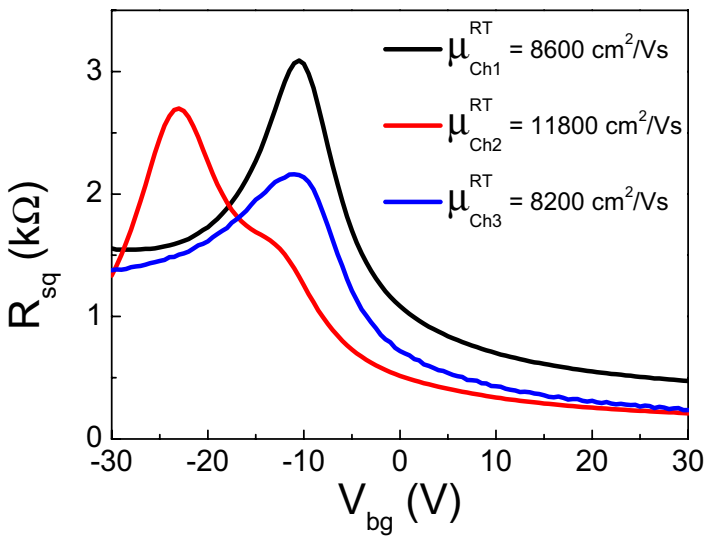

FIG. 1. (a) Schematic of the proposed fully hBN encapsulated graphene spin valve device. (b) An optical microscope image of the fabricated device on $\mathrm{SiO}_{2}(300 \mathrm{~nm}) / \mathrm{Si}$ substrate with multiple ferromagnetic cobalt electrodes $(65 \mathrm{~nm})$ with aluminum capping layer $(4 \mathrm{~nm})$ denoted as $\mathrm{E}(1-6)$. The graphene flake $(0.4 \mathrm{~nm})$ is completely supported underneath by the thick bottom $\mathrm{hBN}(21 \mathrm{~nm})$ flake and encapsulated by the thin-hBN $(0.52 \mathrm{~nm})$ layer between the electrodes E2 and E5. The different regions of the thin-hBN encapsulated graphene channel are denoted as $\mathrm{Ch}(1-4)$ with the double arrows. The boundaries of graphene and thin-hBN flakes are outlined with black and white dashed lines, respectively. (c) Room-temperature square resistance $\left(R_{s q}\right)$ for different graphene channel regions as a function of backgate voltage $\left(V_{b g}\right)$. The corresponding electron mobility values are given in the legend.

E3 and E4) region comprised of graphene encapsulated by the thin $\mathrm{hBN}$. On top of the thin $\mathrm{hBN}$, we put thick top-hBN (6-nm) flake which serves as a topgate dielectric. Finally, $\mathrm{Ch} 4$ (12.5 $\mu \mathrm{m}$, between E2 and E5) consists of region across the whole thin-hBN encapsulated graphene.

The resistance area product $\left(R_{c} A\right)$ for graphene/hBN/ graphene tunnel junction is reported to scale exponentially with the number of hBN layers [14]. We characterize the thin$\mathrm{hBN} /$ graphene interface resistance $\left(R_{c}\right)$ using a three-probe 
measurement scheme. The $R_{c} A$ product for the electrodes $\mathrm{E}(2-5)$ is found to be in the range of $0.3-1.1 \mathrm{k} \Omega \mu \mathrm{m}^{2}$ which agrees with the reported values for single-layer $\mathrm{hBN}$ tunnel barriers $[14,17,18]$. Both the AFM and the $R_{c} A$ analysis confirm the single-layer nature of the thin-hBN flake.

All the measurements are carried out at room temperature in a vacuum of $10^{-6}$ mbar. Charge transport measurements are carried out in a local four-probe measurement scheme. An ac current is passed between electrodes E1 and E6 [Fig. 1(b)], and the voltage drop is measured across the electrodes for different transport channel regions in-between. The measured square resistance $\left(R_{s q}\right)$ is presented in Fig. 1(c) for three different graphene channel regions $\mathrm{Ch} 1, \mathrm{Ch} 2$, and $\mathrm{Ch} 3$. The square resistance for the thin-hBN encapsulated regions [in $\mathrm{Ch}(1-3)$ ] shows charge neutrality point (CNP) around $-12 \mathrm{~V}$ and for the top-hBN encapsulated part of the $\mathrm{Ch} 2$ region show it around $-23 \mathrm{~V}$. A small bump at $-12 \mathrm{~V}$ in $R_{s q}$ data for $\mathrm{Ch} 2$ region corresponds to the thin-hBN encapsulated parts on either side of the top hBN.

The field effect mobility $(\mu)$ of graphene channel is calculated by fitting the $R_{s q}$ data [Fig. 1(c)] using the relation $R_{s q}=1 /\left(n e \mu+\sigma_{0}\right)+\rho_{s}$, where $e$ is the electronic charge, $n$ is the carrier density, $\sigma_{0}$ is the residual conductivity at the CNP, and $\rho_{s}$ is the contribution from short-range scattering [26,27]. The fitted values of the electron mobility for three different regions are $\mu_{\mathrm{Ch} 1}=8600 \mathrm{~cm}^{2} / \mathrm{Vs}, \mu_{\mathrm{Ch} 2}=11800 \mathrm{~cm}^{2} / \mathrm{Vs}$, and $\mu_{\mathrm{Ch} 3}=8200 \mathrm{~cm}^{2} / \mathrm{Vs}$. The close values of mobility at different regions reflect that the graphene channel is homogeneous under the thin-hBN encapsulation. A relatively higher value of mobility for $\mathrm{Ch} 2$ region is attributed to the central top$\mathrm{hBN}$ encapsulated graphene while the remaining thin-hBN encapsulated graphene ( $\mathrm{Ch} 1$ and $\mathrm{Ch} 3$ ) on either side of top $\mathrm{hBN}$ has an uncovered graphene edge of $0.1-0.2 \mu \mathrm{m}$ width which are exposed to polymers during the fabrication.

The spin transport measurements are performed in a fourprobe nonlocal geometry [Fig. 2(a)] at room temperature using standard ac lock-in technique with currents of 10-20 $\mu \mathrm{A}$. We inject a spin polarized current using an ac current source $\left(I_{a c}\right)$ and measure the nonlocal voltage $\left(V_{n l}\right)$ while sweeping an in-plane magnetic field $\left(B_{y}\right)$ parallel to the long axis of the ferromagnetic electrodes. The width of the electrodes is varied from 0.2 to $0.8 \mu \mathrm{m}$ in order to switch magnetization of the electrodes at different coercive fields. As the $B_{y}$ is swept from a negative magnetic field to a positive field, steps in $R_{n l}\left(=V_{n l} / I_{\mathrm{ac}}\right)$ are observed whenever the magnetization of the two inner electrodes changes between parallel and antiparallel configurations. The influence of the outer electrodes will be diminished if chosen far from the injection/detection electrodes, resulting in a typical two-level spin valve signal. In order to assess the spin transport nature of graphene, we extract the spin relaxation parameters from the Hanle spin precession measurements.

Hanle measurements are carried out in a four-probe nonlocal spin valve geometry as shown in Fig. 2(a) while the magnetic field is applied perpendicular $\left(B_{z}\right)$ to the device plane. As the polarization of the injected spins is along the $y$ direction, $B_{z}$ causes the spins to precess in $x-y$ plane with a Larmor frequency $\omega_{L}=g_{e} \mu_{B} B_{z} / \hbar$, where the Lande $g$ factor $g=2, \mu_{B}$ the Bohr magneton, and $\hbar$ the reduced Plank constant. In order to eliminate the background magnetore-

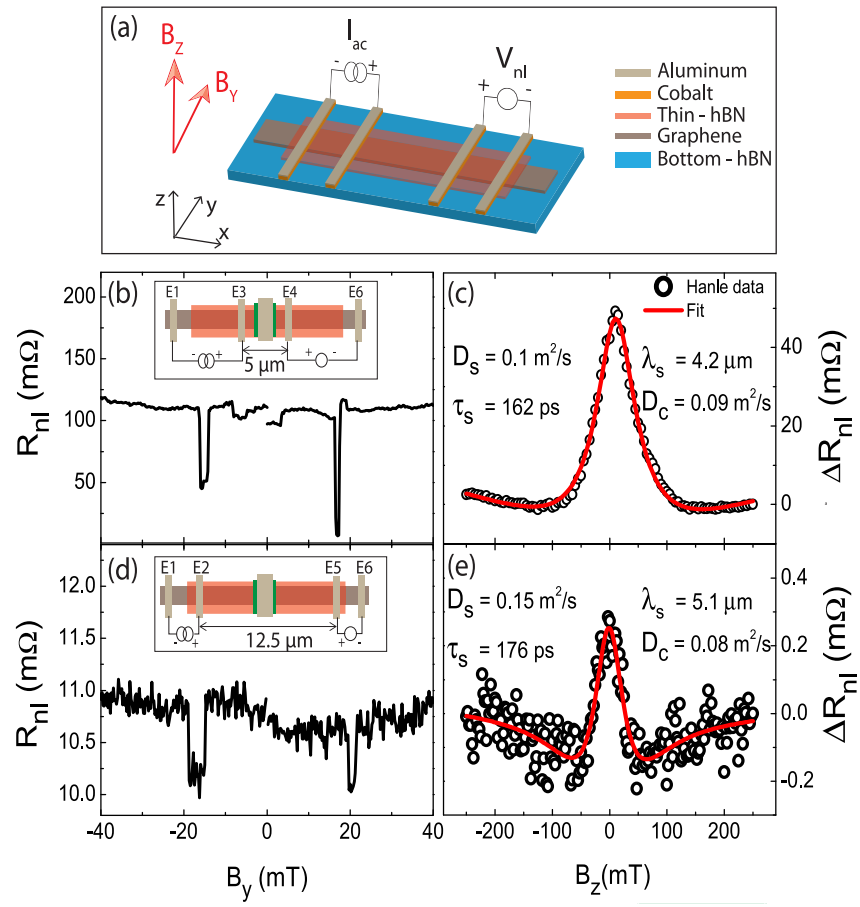

FIG. 2. (a) Schematic of the four-probe nonlocal geometry for the spin valve and the Hanle measurements. Spin valve signals measured at $V_{b g}=30 \mathrm{~V}$ for the top-hBN encapsulated ( $\mathrm{Ch} 2$, between electrodes E3 and E4) region (b), and across the whole thin-hBN encapsulated (Ch4, between electrodes E2 and E5) region (d). The corresponding Hanle signals and fitting curves (c) and (e). The insets in (b) and (d) show the measurement geometry and the length of the graphene channel.

sistance effects, we analyze the effective Hanle spin signal $\Delta R_{n l}=\left(R_{n l}^{\uparrow \uparrow}-R_{n l}^{\uparrow \downarrow}\right) / 2$, where $R_{n l}^{\uparrow \uparrow(\uparrow \downarrow)}$ is the Hanle signal for the parallel (antiparallel) magnetization configuration of the injection and the detection electrodes. The resulting precession data $\Delta R_{n l}$ are fitted with the stationary solutions to the 1D Bloch equation in the diffusion regime; $D_{s} \nabla^{2} \vec{\mu}_{s}-\vec{\mu}_{s} / \tau_{s}+$ $\vec{\omega}_{L} \times \vec{\mu}_{s}=0$, where $\mu_{s}$ is the spin chemical potential, $D_{s}$ is the spin diffusion constant, and $\tau_{s}$ is the spin relaxation time.

The spin valve signals for the $\mathrm{Ch} 2$ region and across the whole thin-hBN encapsulated (Ch4) region are shown in Figs. 2(b) and 2(d), measured at the backgate voltage of $30 \mathrm{~V}$. The corresponding Hanle signals and their fitting curves are shown in Figs. 2(c) and 2(e).

The extracted value of $\tau_{s}$ for different channel regions $[\mathrm{Ch}(1-4)]$ is in the range of $135-176 \mathrm{ps}, D_{s}$ is of $0.11-$ $0.18 \mathrm{~m}^{2} / \mathrm{s}$, and the corresponding value of the spin relaxation length $\lambda_{s}\left(=\sqrt{D_{s} \tau_{s}}\right)$ is of 4.2-5.1 $\mu \mathrm{m}$. The charge diffusion coefficient $\left(D_{c}\right)$ calculated from the resistivity $\left(R_{s q}\right)$ data in Fig. 1(c) is in the range of $0.07-0.09 \mathrm{~m}^{2} / \mathrm{s}$. As the values of $D_{c}$ and $D_{s}$ are in a reasonable agreement, we confirm the reliability of Hanle fitting [22,28].

As we can see from the Hanle fitting data [Figs. 2(c) and $2(e)]$, the spin relaxation parameters do not differ much for different encapsulated regions under thin hBN. Besides, the mobility values also lie close to each other [Fig. 1(c)]. It indicates that a consistent charge and spin transport behavior is observed across different regions of the thin-hBN encapsulated graphene. 
The values of spin relaxation parameters are quite low compared to the graphene on hBN [9] or even partly encapsulated by hBN [20]. The thin-hBN flake is of single layer and it resulted in low interface resistance $\left(R_{c}\right)$ values for the electrodes $\mathrm{E}(2-5), 0.6-2.1 \mathrm{k} \Omega$ which lie in the same order of the spin resistance $\left(R_{\lambda}\right)$ for the thin-hBN encapsulated graphene, $0.4-1.4 \mathrm{k} \Omega$ where $R_{\lambda}=R_{s q} \lambda_{s} / W, W=1.8 \mu \mathrm{m}$ is the width of the graphene. These values imply that the device is within the conductivity mismatch regime [28]. A similar behavior is also reported by Yamaguchi et al. [16] with exfoliated 1L-hBN and Fu et al. [18] with CVD 1L-hBN tunnel barriers.

It is also important to note in our device that the ferromagnetic cobalt electrodes on top of the thin-hBN layer are deposited in order to avoid contact with the uncovered (by thin-hBN) graphene edge [Fig. 1(b)]. The proximity of the stray magnetic field from the ends of the cobalt electrodes (between E2 and E5) can act as an additional dephasing field and influence the spin transport in graphene. Also, the uncovered region is exposed to the PC and PMMA polymers during the fabrication which can reduce the mobility and spin relaxation time.

Further, we would like to emphasize that the spin diffusion is detected across $12.5-\mu \mathrm{m}$ length of thin-hBN encapsulated (Ch4) graphene region [Figs. 2(d) and 2(e)] which consists of multiple electrodes in-between the spin injection (E2) and the spin detection (E5) electrodes [Fig. 1(b)]. The conventional oxide-barrier/graphene interfaces are reported to act as spin sinks especially when their $R_{c}$ lie close to $R_{\lambda}[12,28,29]$ which hinders the long distance spin transport in graphene with multiple electrodes. Whereas, within the conductivity mismatched regime, our device still performs better than the device with oxide barriers, which might have pinholes. Hence, we attribute the observed long distance spin transport behavior to the pinhole-free nature of the thin-hBN layer and to the clean interface of thin $\mathrm{hBN}$ with the graphene compared to the deposited oxide tunnel barrier.

We would also like to point out that we do not observe the very long spin relaxation length or high spin relaxation time as in the case of partly hBN encapsulated graphene stack reported by Guimarães et al. [20], and it is worthwhile to mention the differences between our device and the stack reported in Ref. [20]. In our device, PMMA residues lie at the thin-hBN/cobalt electrode interface which might affect the spin injection/detection in a different way than in the stack reported in Ref. [20], which has PMMA residues between the graphene and the oxide tunnel barrier. Further, our device shows lower mobility and charge diffusion constant which also translates to the lower spin relaxation length.

Furthermore, using our device structure we can control the electric field and the carrier density independently with the top and the bottom gate electrodes. This allows us to study (i) the electrical control of spin information in graphene [20], and (ii) the spin transport across the $p / n$ junctions created by the topgate and the nontopgate encapsulated graphene. It is interesting to study the spin transport across the $p / n$ junction, which acts as a barrier for the transmission of spins, and results in high magnetoresistance and sensitivity in a spin valve transistor [30].
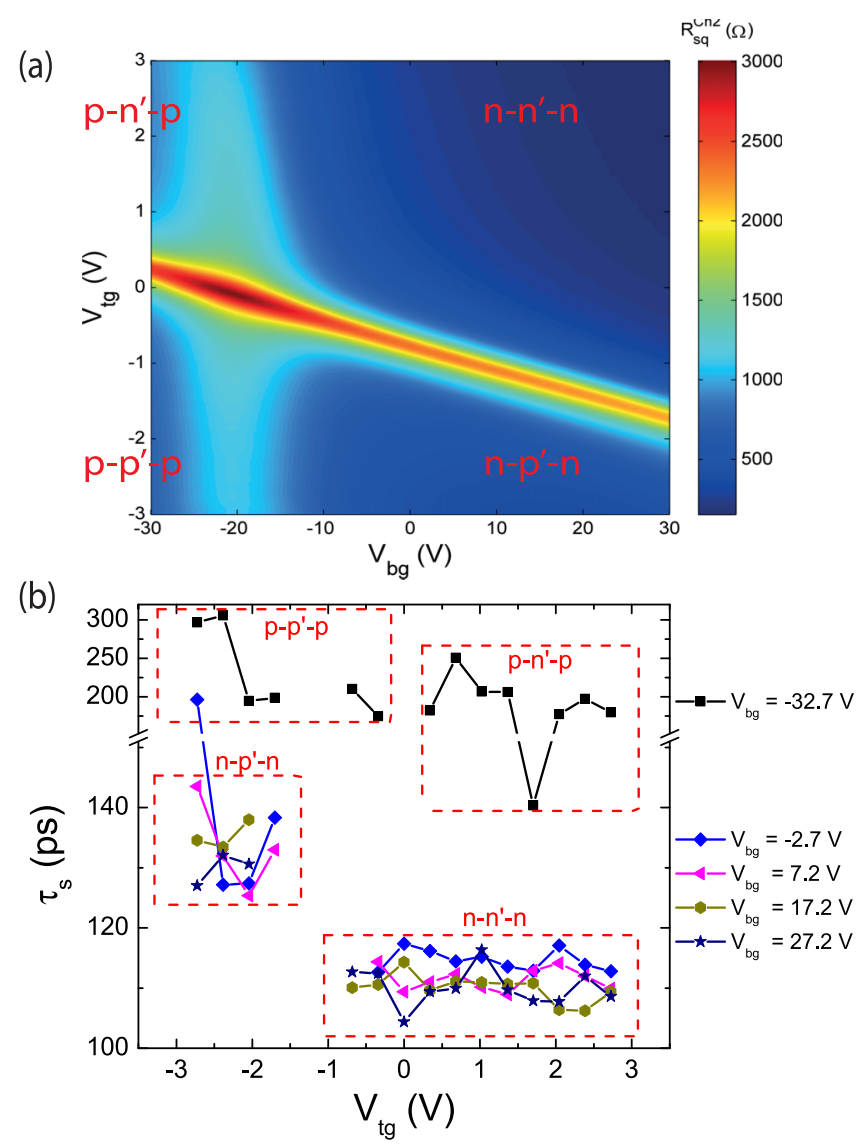

FIG. 3. (a) Contour plot of square resistance $R_{s q}$ for top-hBN encapsulated $(\mathrm{Ch} 2)$ region measured at room temperature with respect to backgate $\left(V_{b g}\right)$ and topgate $\left(V_{t g}\right)$ voltages. The polarities $p$ or $n\left(p^{\prime}\right.$ or $\left.n^{\prime}\right)$ type of nontopgated (topgated) encapsulated regions are tuned independently using the combinations of $V_{b g}$ and $V_{t g}$ which resulted in $n-n^{\prime}-n, n-p^{\prime}-n, p-n^{\prime}-p$, and $p-p^{\prime}-p$ junction configurations. (b) Spin relaxation time as a function of topgate voltage at different backgate voltages. The dashed red lines outline the data corresponding to the four junction configurations.

A contour plot for the square resistance of the $\mathrm{Ch} 2$ region $\left(R_{s q}^{\mathrm{Ch} 2}\right)$ as a function of topgate voltage $V_{t g}$ and backgate voltage $V_{b g}$ is shown in Fig. 3(a). The charge neutrality point (CNP) appears as a line with a slope of -0.034 which is equivalent to the ratio of the top and bottom gate dielectric capacitances. The maximum $R_{s q}$ of the CNP appears at $\mathrm{V}_{b g} \approx-22.7 \mathrm{~V}, V_{t g} \approx 0 \mathrm{~V}$ and decreases along either side of the line. It is a characteristic behavior observed with single-layer graphene [20]. The $V_{t g}$ independent feature around $\mathrm{V}_{b g}=-22.7 \mathrm{~V}$ appears due to the sides of the top-hBN encapsulated region that are nontopgated between the electrodes E3 and E4 [21].

We can create an effective electric field in the top-hBN encapsulated part of the $\mathrm{Ch} 2$ region by modulating the topgate voltage and the backgate voltage [31]. The perpendicular electric field can induce a Rashba spin orbit field in graphene [20] which can be used to manipulate the spin transport properties of the topgate encapsulated graphene channel.

The maximum value of the electric field created within the range of applied $V_{t g}$ and $V_{b g}$ is $0.22 \mathrm{~V} / \mathrm{nm}$. We do not observe a significant dependence of the spin relaxation time on the 
electric field. We attribute this to the lower field compared to the Rashba field $(0.7 \mathrm{~V} / \mathrm{nm})$ applied in the case of Guimarães et al. [20].

Within the $\mathrm{Ch} 2$ region, carrier density in the nontopgate encapsulated parts is controlled by $V_{b g}$ whereas the central topgate encapsulated part is controlled by both $V_{b g}$ and $V_{t g}$. This clearly resulted in four quadrants representing different $p / n$ junction configurations [32] as indicated in Fig. 3(a). Due to the novelty of our device fabrication we could see four quadrants compared to the similar geometry reported in Refs. [20,21]. The nontopgated graphene regions in Refs. [20,21] are highly $n$ doped by the polycarbonate. However, in our case, graphene in the topgated region as well as the nontopgated region is protected by the thin $\mathrm{hBN}$ and is not doped by polymers. So, we are also able to access the $p$-doped characteristics of the nontopgated regions.

A strong dependence of the spin relaxation time $\left(\tau_{s}\right)$ among the four junction configurations is observed as shown in Fig. 3(b). On average, the relaxation time is increased when the $n\left(n^{\prime}\right)$-doped region changes to $p\left(p^{\prime}\right)$-doped region. This can be observed at different topgate voltages as we move from $n-n^{\prime}-n$ region to $n-p^{\prime}-n$ region, and further to $p-p^{\prime}-p$ region. A possible explanation for the observed behavior is that the contacts can induce a slight doping, as can be seen from the asymmetric square resistance of graphene in Fig. 1(c). This might result in an additional $p$ - $n$ interface in the $p$-doped regime, decoupling the contacts from the channel and resulting in higher spin relaxation time for holes. Furthermore, the gate dependence of spin lifetime in Fig. 3(b) does not show a dip around the CNP [20,33] possibly due to the influence of $p-n$ junctions formed at the edges of the topgated graphene channel near the CNP.

\section{CONCLUSIONS}

In conclusion, we demonstrate a fully hBN encapsulated graphene device for spintronics applications. We show that the full encapsulation of graphene results in homogeneous charge and spin transport properties at room temperature. Charge transport measurements show uniform mobility across different regions of the encapsulated graphene. Spin transport measurements show that a uniform spin relaxation length across different channel regions is achieved with the crystalline hBN encapsulation. Further, our device shows spin transport across the whole thin-hBN encapsulated region of $12.5-\mu \mathrm{m}$ length even in the presence of conductivity mismatch electrodes, demonstrating the potential of using $\mathrm{hBN}$ as a tunnel barrier for graphene spintronics. The dual gate geometry allowed us to study the effect of electric field on the spin transport. However, we do not observe a significant dependence due to the low values of achieved electric field. Moreover, we observe a strong dependence of the spin relaxation time on different $p / n$ junction configurations. Further investigation is necessary to explain this behavior.

\section{ACKNOWLEDGMENTS}

We kindly acknowledge J. G. Holstein, H. M. de Roosz, H. Adema, and T. J. Schouten for the technical assistance. We thank J. Ingla-Aynés for help in sample preparation and for the fruitful discussions. The research leading to these results has received funding from the European Union Seventh Framework Programme under Grant Agreement No. 604391 Graphene Flagship and supported by the Zernike Institute for Advanced Materials and the Nederlandse Organisatie voor Wetenschappelijk (NWO).
[1] A. H. Castro Neto, F. Guinea, N. M. R. Peres, K. S. Novoselov, and A. K. Geim, Rev. Mod. Phys. 81, 109 (2009).

[2] S. Das Sarma, S. Adam, E. H. Hwang, and E. Rossi, Rev. Mod. Phys. 83, 407 (2011).

[3] V. K. Dugaev, E. Y. Sherman, and J. Barnaś, Phys. Rev. B 83, 085306 (2011).

[4] H. Min, J. E. Hill, N. A. Sinitsyn, B. R. Sahu, L. Kleinman, and A. H. MacDonald, Phys. Rev. B 74, 165310 (2006).

[5] D. Pesin and A. H. MacDonald, Nat. Mater. 11, 409 (2012).

[6] M. Drogeler, C. Franzen, F. Volmer, T. Pohlmann, L. Banszerus, M. Wolter, K. Watanabe, T. Taniguchi, C. Stampfer, and B. Beschoten, arXiv:1602.02725.

[7] S. Roche and S. O. Valenzuela, J. Phys. D: Appl. Phys. 47, 094011 (2014).

[8] L. Wang, I. Meric, P. Y. Huang, Q. Gao, Y. Gao, H. Tran, T. Taniguchi, K. Watanabe, L. M. Campos, D. A. Muller, J. Guo, P. Kim, J. Hone, K. L. Shepard, and C. R. Dean, Science 342, 614 (2013).

[9] P. J. Zomer, M. H. D. Guimaraes, N. Tombros, and B. J. van Wees, Phys. Rev. B 86, 161416 (2012).

[10] D. Van Tuan, F. Ortmann, A. W. Cummings, D. Soriano, and S. Roche, Sci. Rep. 6, 21046 (2016).
[11] A. V. Kretinin, Y. Cao, J. S. Tu, G. L. Yu, R. Jalil, K. S. Novoselov, S. J. Haigh, A. Gholinia, A. Mishchenko, M. Lozada, T. Georgiou, C. R. Woods, F. Withers, P. Blake, G. Eda, A. Wirsig, C. Hucho, K. Watanabe, T. Taniguchi, A. K. Geim, and R. V. Gorbachev, Nano Lett. 14, 3270 (2014).

[12] F. Volmer, M. Drögeler, E. Maynicke, N. von den Driesch, M. L. Boschen, G. Güntherodt, C. Stampfer, and B. Beschoten, Phys. Rev. B 90, 165403 (2014).

[13] G.-H. Lee, Y.-J. Yu, C. Lee, C. Dean, K. L. Shepard, P. Kim, and J. Hone, Appl. Phys. Lett. 99, 243114 (2011).

[14] L. Britnell, R. V. Gorbachev, R. Jalil, B. D. Belle, F. Schedin, M. I. Katsnelson, L. Eaves, S. V. Morozov, A. S. Mayorov, N. M. R. Peres, A. H. C. Neto, J. Leist, A. K. Geim, L. A. Ponomarenko, and K. S. Novoselov, Nano Lett. 12, 1707 (2012).

[15] Q. Wu, L. Shen, Z. Bai, M. Zeng, M. Yang, Z. Huang, and Y. P. Feng, Phys. Rev. Appl. 2, 044008 (2014).

[16] T. Yamaguchi, Y. Inoue, S. Masubuchi, S. Morikawa, M. Onuki, K. Watanabe, T. Taniguchi, R. Moriya, and T. Machida, Appl. Phys. Express 6, 073001 (2013).

[17] M. V. Kamalakar, A. Dankert, J. Bergsten, T. Ive, and S. P. Dash, Sci. Rep. 4, 6146 (2014). 
[18] W. Fu, P. Makk, R. Maurand, M. Bräuninger, and C. Schönenberger, J. Appl. Phys. 116, 074306 (2014).

[19] P. J. Zomer, M. H. D. Guimares, J. C. Brant, N. Tombros, and B. J. v. Wees, Appl. Phys. Lett. 105, 013101 (2014).

[20] M. H. D. Guimaraes, P. J. Zomer, J. Ingla-Aynes, J. C. Brant, N. Tombros, and B. J. van Wees, Phys. Rev. Lett. 113, 086602 (2014).

[21] J. Ingla-Aynés, M. H. D. Guimarães, R. J. Meijerink, P. J. Zomer, and B. J. van Wees, Phys. Rev. B 92, 201410 (2015).

[22] M. H. D. Guimares, A. Veligura, P. J. Zomer, T. Maassen, I. J. Vera-Marun, N. Tombros, and B. J. van Wees, Nano Lett. 12, 3512 (2012).

[23] P. Blake, E. W. Hill, A. H. Castro Neto, K. S. Novoselov, D. Jiang, R. Yang, T. J. Booth, and A. K. Geim, Appl. Phys. Lett. 91, 063124 (2007).

[24] R. V. Gorbachev, I. Riaz, R. R. Nair, R. Jalil, L. Britnell, B. D. Belle, E. W. Hill, K. S. Novoselov, K. Watanabe, T. Taniguchi, A. K. Geim, and P. Blake, Small 7, 465 (2011).

[25] N. Tombros, C. Jozsa, M. Popinciuc, H. T. Jonkman, and B. J. van Wees, Nature (London) 448, 571 (2007).
[26] S. V. Morozov, K. S. Novoselov, M. I. Katsnelson, F. Schedin, D. C. Elias, J. A. Jaszczak, and A. K. Geim, Phys. Rev. Lett. 100, 016602 (2008).

[27] P. J. Zomer, S. P. Dash, N. Tombros, and B. J. van Wees, Appl. Phys. Lett. 99, 232104 (2011).

[28] T. Maassen, I. J. Vera-Marun, M. H. D. Guimarães, and B. J. van Wees, Phys. Rev. B 86, 235408 (2012).

[29] W. Han, K. Pi, K. M. McCreary, Y. Li, J. J. I. Wong, A. G. Swartz, and R. K. Kawakami, Phys. Rev. Lett. 105, 167202 (2010).

[30] Y. W. Huang, C. K. Lo, Y. D. Yao, L. C. Hsieh, and J. H. Huang, J. Appl. Phys. 97, 10D504 (2005).

[31] Y. Zhang, T.-T. Tang, C. Girit, Z. Hao, M. C. Martin, A. Zettl, M. F. Crommie, Y. R. Shen, and F. Wang, Nature (London) 459, 820 (2009).

[32] J. R. Williams, L. DiCarlo, and C. M. Marcus, Science 317, 638 (2007).

[33] W. Han and R. K. Kawakami, Phys. Rev. Lett. 107, 047207 (2011). 